Research Article

\title{
Investigation and Control of the Blasting-Induced Ground Vibration under Cold Condition
}

\author{
Yingguo Hu, Zhaowei Yang ${ }^{D}$, Erlei Yao, Meishan Liu, and Yu Rao \\ Key Laboratory of Geotechnical Mechanics and Engineering of Ministry of Water Resources, \\ Changjiang River Scientific Research Institute, Wuhan 430010, Hubei, China \\ Correspondence should be addressed to Zhaowei Yang; yangzw@whu.edu.cn
}

Received 29 December 2020; Revised 12 February 2021; Accepted 23 March 2021; Published 8 April 2021

Academic Editor: Nan Jiang

Copyright (c) 2021 Yingguo Hu et al. This is an open access article distributed under the Creative Commons Attribution License, which permits unrestricted use, distribution, and reproduction in any medium, provided the original work is properly cited.

This paper focuses on the investigation and control of the blasting-induced ground vibration under cold condition. The mechanical performance and wave propagation characteristics of the frozen rock mass are quite different from that of the conventional condition. Laboratory tests were implemented to investigate the wave impedance of rock mass in the frozen, saturated, normal, and drying states. Results reveal the longitudinal wave velocity could be enlarged by 40 percent in the frozen state. Then long-term monitoring of blasting vibration was implemented based on the blasting excavation of the Fengman hydropower station reconstruction project in the north of China. Results demonstrate the PPV and frequency both attenuate much slower when the rock mass is frozen, and the obvious turning points of PPV could be found between different temperatures, where the change of the PPV relationship happens. At last, numerical simulation of the blasting seismic wave attenuation and the response in the protected structure was implemented. The equivalent freezing simulation method was proposed and verified with the site experiment data. Results demonstrate that the attenuation coefficient decreases obviously as the frozen depth of the rock mass increases. The dynamic degree response in structure is much stronger and the maximum charge weight per delay was limited more strictly under the frozen condition. A most adverse frozen depth was determined when the charge weight per delay gets the minimum value. With the above control approaches, a total of 676 blasting was completed in Fengman hydropower station reconstruction and no case of excessive measurement could be found.

\section{Introduction}

Blasting is often a necessary part of mining and construction operations and is the most cost-effective way to break rock. When an explosive is detonated, a chemical reaction occurs very rapidly and a relatively small quantity of explosive is converted into gas of very high temperature. This reaction results in two types of loadings applied on the borehole wall, namely, a stress wave and a gas pressure with longer duration [1-4]. The detonation properties of the explosive consist of the explosion pressure, its time history, and the total energy delivered to the rock. The response of the rock mass to such time-varying high-amplitude stresses is even more complex, as all the relevant strain-rate-dependent properties of the subject rock are not known. For this case, it is essential to implement both experimental study and numerical study. The experimental study could generate an experimental database, and the numerical study could simulate the processes of blasting and fragmentation through the use of numerical models so as to obtain a better understanding of the dominant parameters that control blast results [5-9].

In a properly designed blast, the major part of the explosion energy is used in crushing and breaking the rock. However, some energy also causes noise and ground vibration. The prediction of ground vibration components has great importance in the minimization of environmental complaints $[10,11]$. Estimating the particle velocity and other components of ground vibration with reliable approaches will be very useful in blast design. The most widely used method is the so-called "scaled distance" method, based on the empirical principle that states peak particle velocity 
(PPV) at a point is inversely proportional to the distance from the blast and shows a square root dependence on the maximum charge per delay [12-16]. There are many ground motion predictors proposed by different authors based on their own collection of data from different sites. But, due to the complexity of the geological and technological and other associated parameters in blasting, a prediction of PPV is difficult and sometimes confusing if these attenuation equations are not updated due to the movement of blasting places [17-20].

The existed literature studies demonstrate that blasting vibration attenuation law is quite related to the physical and mechanical parameters of the rock mass. Since the structures are surrounded by jointed rock mass, the blast wave propagation in the rock mass is significantly influenced by the joints $[21,22]$. The vastly existed joints in rock mass not affect only the mechanical properties of the rock mass but also their dynamic response. The water usually exists in the joint, but it could turn into ice under the low temperature. The wave impedance of the ice is quite different from that of water, which significantly affects the vibration attenuation. This is a concern problem during the blasting excavation in the cold region which has a large temperature difference in summer and winter. However, there is still some lack of knowledge about the blasting vibration control for the rock blasting excavation under cold condition.

This paper focuses on the blasting earthquake response of reconstruction project under cold condition. Laboratory tests were implemented to investigate the change of mechanical performance for rock mass at the frozen, saturated, normal, and drying states. Then long-term monitoring of blasting vibration was implemented based on the blasting excavation of Fengman hydropower station reconstruction project in China to study the vibration attenuation law of the rock mass at different seasons. At last, numerical simulation of the blasting seismic wave attenuation and the response in the protected structure at different states was finished and suggestion of blasting earthquake control of rock mass excavation under cold condition was proposed.

\section{Laboratory Study on Physical Properties of the Rock Mass under Freezing}

When the rock is frozen, the propagation medium of the seismic wave is changed from the water-rock combination solid to the ice-rock combination solid. In general, the water density is $1000 \mathrm{~kg} / \mathrm{m}^{3}$, and the P-wave velocity is $1500 \mathrm{~m} / \mathrm{s}$, while the ice density is $900 \mathrm{~kg} / \mathrm{m}^{3}$, the P-wave velocity is about $4500 \mathrm{~m} / \mathrm{s}$. The wave impedance of the ice is about three times that of water. Therefore, the propagation of seismic waves in frozen rock could be quite different from that of the conventional condition. In the next section, the laboratory experiment was implemented to investigate the physical and mechanical properties of the rock mass under frozen state.

2.1. Experiment Design. In the present study, the laboratory experiment includes two important aspects: the first step is measuring the sonic wave velocity of different kinds of rock medium in different states. By controlling the temperature and humidity of the air, the rock mass could be in the state of dry, natural, saturated, and frozen conditions. The rock medium of sandstone, mudstone, glutenite, limestone, marble, basalt, dolomite, and granite was selected. The second step is investigating the change of the density and sonic wave velocity in the sandstone of different porosities under frozen state. The porosity of $5 \% \sim 30 \%$ was selected to be studied in the experiment. Figure 1 plots a photograph of the frozen sandstone specimen and equipment of the sonic wave test.

\subsection{Comparison of the Physical Properties of Rock Medium} under Different States. The waveform of the sonic wave test could reflect the damping characteristic of the rock mass according to the existed literature studies. When the rock mass is of better quality, the starting point of the longitudinal wave is more obvious and the frequency of the sonic waveform is higher. Figure 2 plots the comparison of the waveform of the sonic wave test for sandstone under conventional and frozen states.

It can be seen that the starting point of the longitudinal wave is much clearer in the frozen state than in the conventional state. The amplitude of the longitudinal wave in the frozen sandstone at the start point is about ten times that in the conventional rock mass. The frequency of frozen state for sandstone is also much larger than that of conventional state, while the former is about five times the latter. Results demonstrate that the waveform of the sonic wave test for the sandstone in the frozen state is more close to that of the rock mass of better quality, which reveals that the damping property of the rock is weakened after frozen. Figure 3 plots the comparison of longitudinal wave velocity of different kinds of rock medium in different states.

The longitudinal wave velocity of the rock mass in the frozen state is the biggest, and the wet saturated state takes the second place, while the natural state is third and the dry state is least. It can be explained that when the rock is frozen, the liquid water in the joint changes into solid ice, which could improve the propagation of the longitudinal wave. But for the rock medium in the dry state, the water was vaporized by the wind, and the air was filled with joints. It is known that the longitudinal wave velocity in the ice is larger than that in water, but the longitudinal wave velocity in water exceeds that in the air by 2 times. The difference between the longitudinal wave velocity in the frozen and natural states is about $30 \%$ in the sandstone, but in the granite that is only about $5 \%$. That is because the granite is usually dense, and the porosity is less than that of sandstone, which weakens the change of physical properties when the rock mass is frozen. Figure 4 plots the comparison of longitudinal wave velocity of sandstone of different porosities in the frozen and natural states.

Results reveal that as the porosity increases, the longitudinal wave velocity decreases obviously, and the longitudinal wave velocity and the porosity obey the linear law. When the rock mass is frozen, the longitudinal wave velocity exceeds that of the natural state by about $10 \% \sim 35 \%$. The 


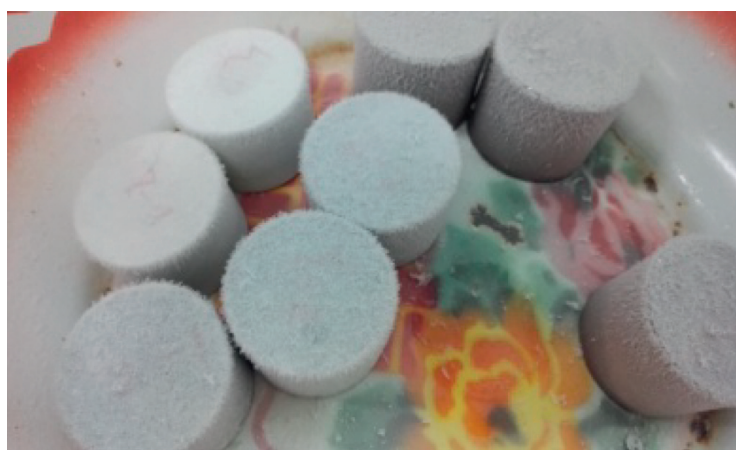

(a)

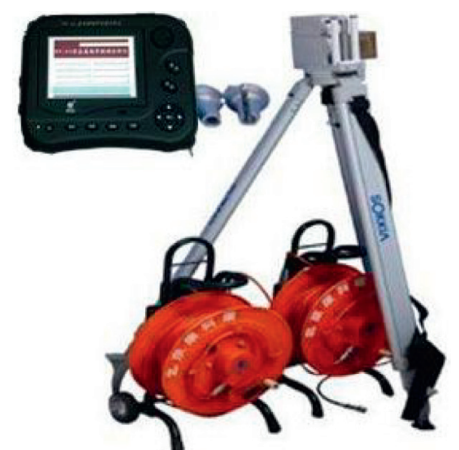

(b)

FIgURE 1: Photograph of the (a) frozen sandstone specimen and (b) equipment of the sonic wave test.

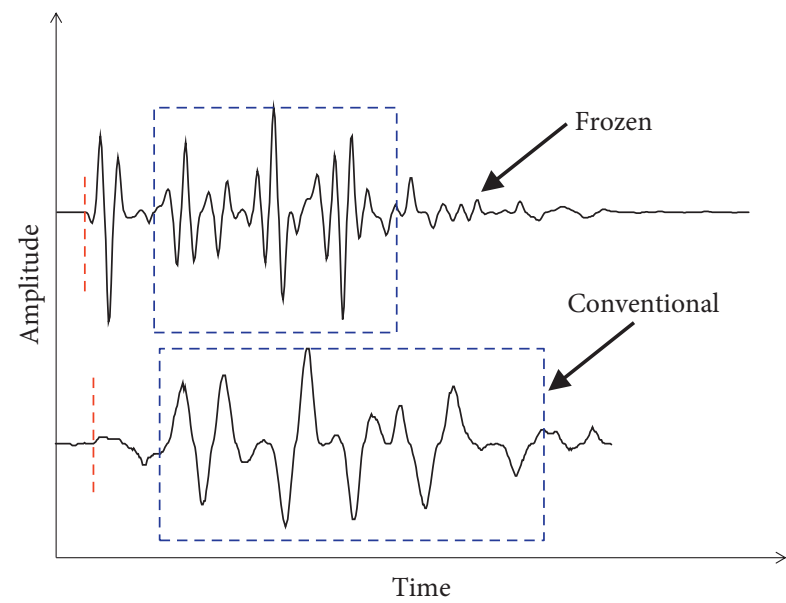

Figure 2: Comparison of the waveform of the sonic wave test under conventional and frozen states.

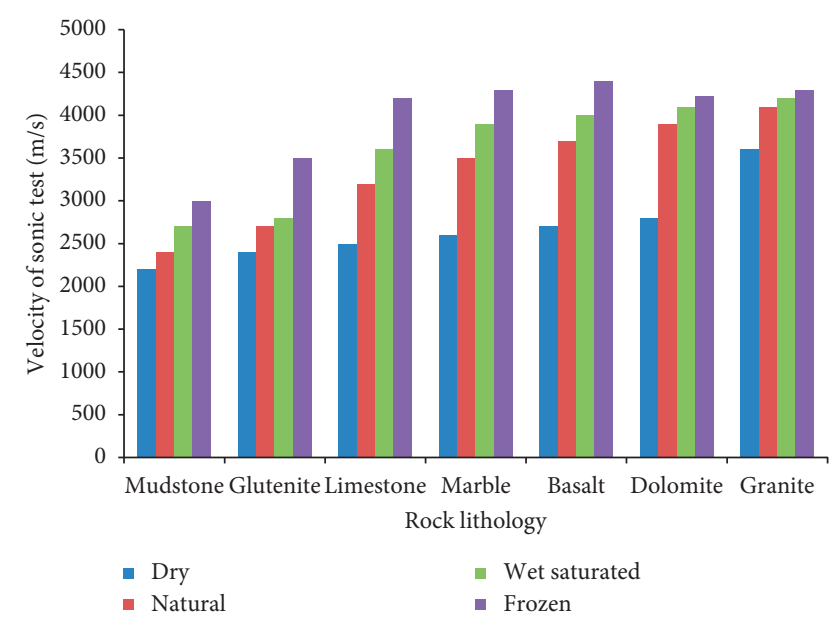

FIGURE 3: Comparison of longitudinal wave velocity of different kinds of rock medium in different states.

difference between the frozen state and the natural state increases with the porosity obviously. That is because as the porosity increases, the effect proportion of water changing

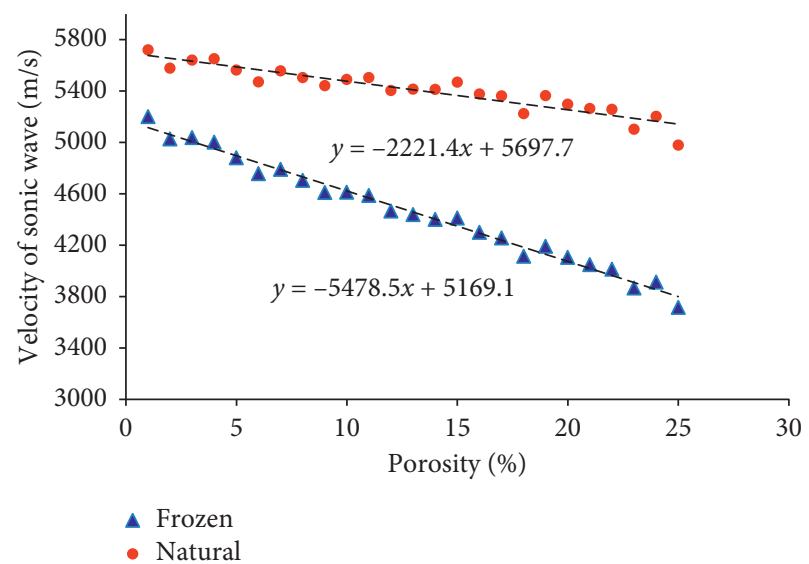

Figure 4: Comparison of longitudinal wave velocity of sandstone of different porosities in the frozen and natural states.

into ice is enlarged, which makes the longitudinal wave velocity in the frozen rock mass larger. It is known that wave impedance is a very important index in evaluating the propagation of stress wave in the rock mass. Figure 5 plots the curve of wave impedance increment versus the porosity when the sandstone is in the frozen state.

It can be seen that as the porosity increases, the enlargement of wave impedance increment could be found. The wave impedance increment and the porosity obey the exponential relationships obviously, while the coefficient of correlation reaches as much as 0.88 . Results demonstrate that the ability to conduct the stress waves is enlarged when the rock mass is frozen, especially in the rock medium with high porosity. But it should be pointed that it is not a complete good change during the blasting vibration control process. Firstly, when the rock mass is frozen, and the physical properties of the rock mass become better, the attenuation of blasting stress wave is slower, which is not helpful for the safety of the protective structures in the far field. Secondly, the frozen depth is usually about $0.5 \sim 3 \mathrm{~m}$ in the engineering practice, the rock is layered in the frozen state, which makes the propagation of the stress wave more complex, and it is difficult to determine reasonable blasting parameters under this condition. 


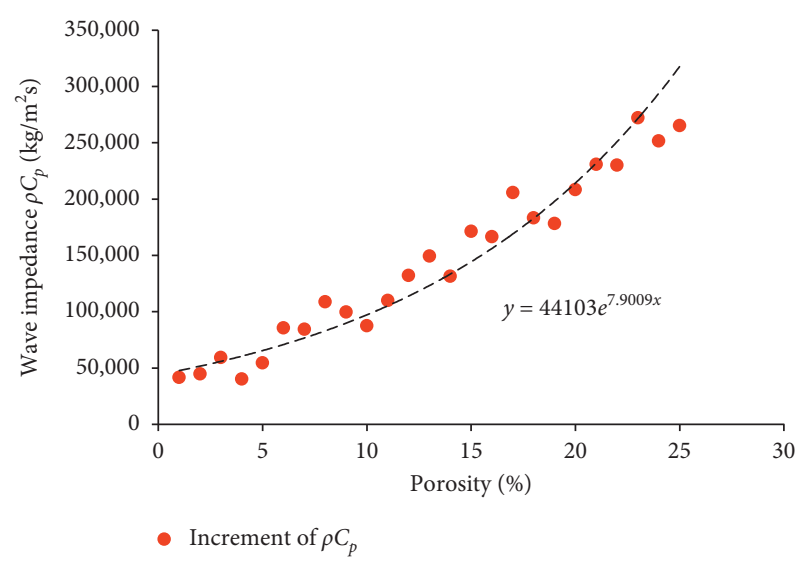

FIGURE 5: Wave impedance increment versus the porosity in the frozen sandstone.

\section{Field Experiment of the Blasting Vibration Attenuation Law in Cold Condition}

3.1. Project Ground. Field experiments were implemented to study the blasting vibration attenuation law in cold condition based on the foundation excavation of the Fengman hydropower station. Fengman hydropower station is one of the most famous hydropower projects in China. It was built in 1937 in the Songhua River in Jilin province. The Chinese government decides to implement the reconstruction to increase installed capacity in 2012, and the protection of the existing buildings in the hydropower station was one of the most concern problems during the blasting excavation of reconstruction. Another difficulty is that the temperature in the Fengman hydropower station is very low in autumn and winter. Figure 6 shows the photograph of the engineering site. The annual development curve of the temperature and frozen depth of the rock mass is plotted in Figure 7.

It can be seen that the temperature at the Fengman hydropower station in five months is under $0^{\circ} \mathrm{C}$, which means the rock mass is in the frozen state for about a half year. According to meteorological data, the frozen depth of the rock mass is between 0.25 and $2.25 \mathrm{~m}$. The rock lithology of the Fengman hydropower project foundation is sandstone. With the results of the laboratory test, the physical properties of sandstone in the frozen state are quite different from the natural state. So the field experiment is implemented in this section to study the blasting vibration attenuation law in cold condition. The design of the experiment is measuring the blasting vibration at different detonation distances, and the measurement was implemented at different season and temperature conditions. Figure 8 shows the typical layout of the vibration measurement points, the blasting vibration equipment (Minimate Plus), and the typically measured blasting vibration waveform. The measurement range of this instrument is $0.001 \sim 25.4 \mathrm{~cm} / \mathrm{s}$. The sampling rate was taken as $125 \mu$ s, i.e. recording 8000 samples per second.

3.2. Comparison of Blasting Vibration in Cold and Conventional Conditions. To investigate the blasting vibration characteristic under different temperatures, the comparison

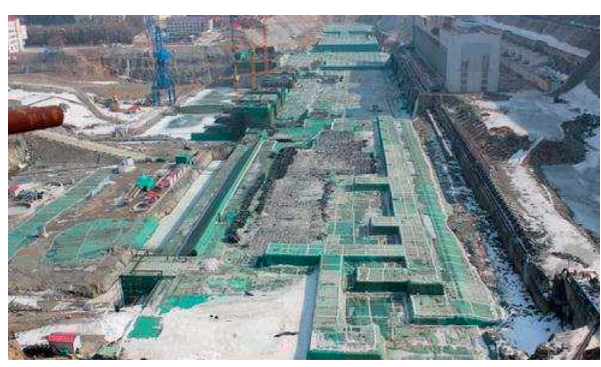

FIgURE 6: Photograph of engineering site of Fengman hydropower station.

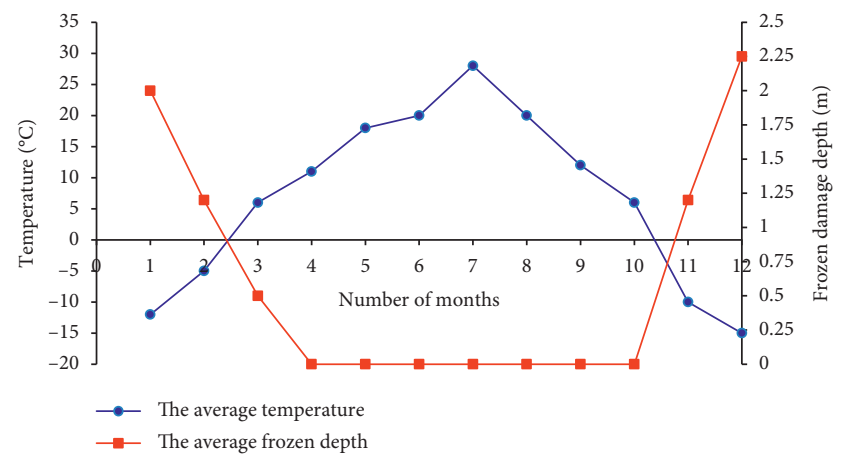

Figure 7: The annual development curve of the temperature in a year.

of the curve of blasting vibration and frequency was implemented. To ensure the effectiveness of the results, the charge weight, detonation distance, and blasting vibration equipment are all the same. Figure 9 plots two comparison examples of blasting vibration waveform in the conventional and frozen rock mass.

It can be seen that the PPV of the frozen rock mass is larger than that of the conventional rock mass. When the detonation distance is $15 \mathrm{~m}$, the PPV in the frozen rock mass exceeds that under conventional condition by $0.9 \mathrm{~cm} / \mathrm{s}$. But the difference of PPV increases when the detonation distance is $20 \mathrm{~m}$, in which the PPV under frozen condition exceeds that of the conventional rock mass with $2.2 \mathrm{~cm} / \mathrm{s}$. The frequency of the blasting vibration in the frozen rock mass is also larger than that in conventional condition. The dominant frequency of blasting vibration in the conventional condition is about $110 \mathrm{~Hz}$, while that is $289 \mathrm{~Hz}$ after the rock mass is frozen. The increment rate of dominant frequency is about $171 \%$. Results demonstrate that the PPV and the dominant frequency were both enlarged after the rock mass is frozen, which indicates that the attenuation speed of blasting seismic wave is slower. Figure 10 plots the distribution of PPV versus detonation distance at the temperature of $-5^{\circ} \mathrm{C}$ and $-20^{\circ} \mathrm{C}$.

The turning point could be found obviously during the comparison of PPV at different detonation distances. When the detonation distance is less than the turning point, the PPV of the rock mass under $20^{\circ} \mathrm{C}$ is larger than that under $-5^{\circ} \mathrm{C}$, but when the detonation distance is larger than the turning point, the relationship is opposite. During engineering practice, the buildings which need to be protected 


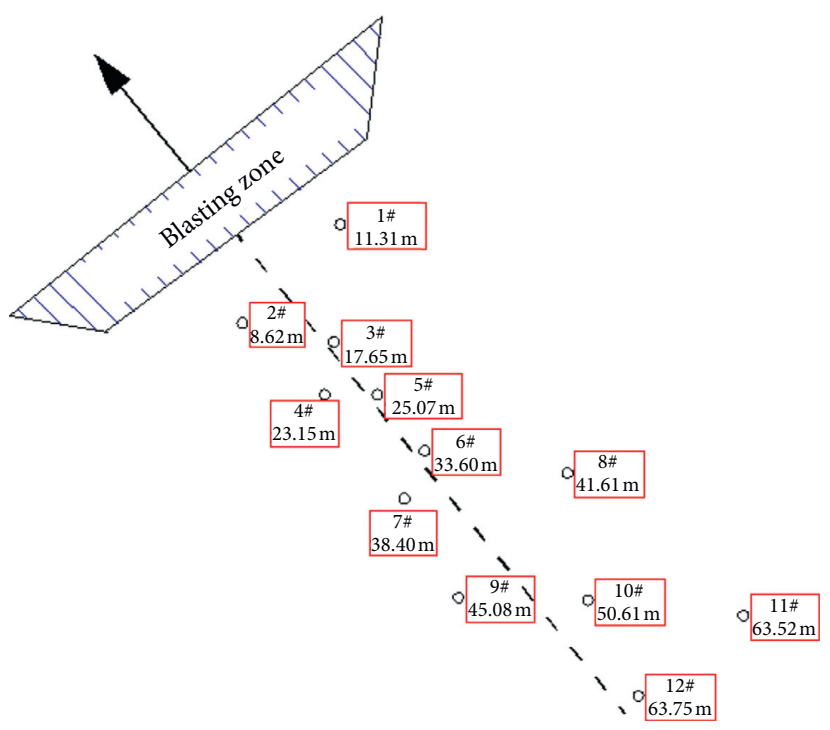

(a)

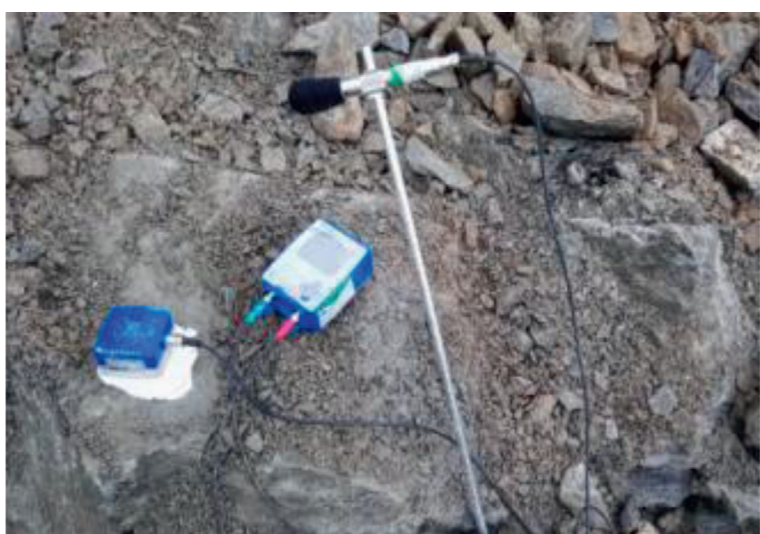

(b)

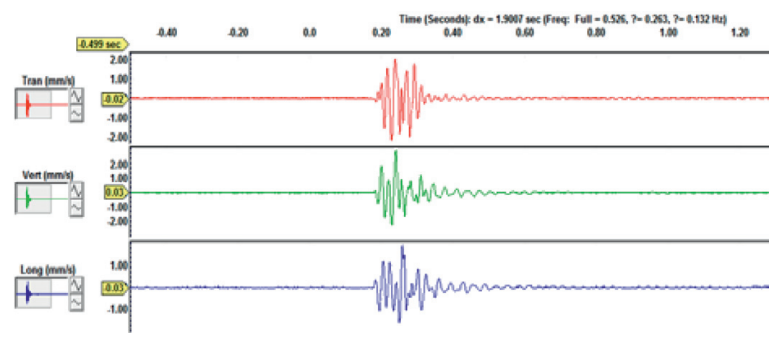

(c)

Figure 8: The design, equipment, and typical waveform of the site monitoring. (a) Typical layout of measurement. (b) Equipment of blasting vibration. (c) Typical waveform.

are usually located in different detonation distances. Results reveal that when the protected objects are located in the position close to the blast hole, it is dangerous when the rock mass is in the state of $20^{\circ} \mathrm{C}$, but if the protected objects are farther than the turning point, the temperature of $-5^{\circ} \mathrm{C}$ is not helpful for safety. It can be seen that the temperature state of the rock mass could affect the structure security control of blasting vibration significantly, which is rarely noticed in the existed literature.

\subsection{Comparison of PPV Attenuation Law at Different} Temperatures. Estimating the particle velocity and other components of ground vibration with reliable approaches will be very useful in blast design. The most widely used method is the so-called "scaled distance" method, based on the empirical principle that states peak particle velocity (PPV) at a point is inversely proportional to the distance from the blast and shows a square root dependence on the maximum charge per delay. Figures 11 and 12 plot the PPV at different scaled distances of the $Y$ direction in the rock mass under different temperatures.

It can be seen that the relationship between the PPV and scaled distances is clearly because the correlation coefficients exceed 0.9. The attenuation coefficients are quite different when the temperature of the rock mass varies. As the temperature decreases, the attenuation coefficient $K$ and $\alpha$ all decrease by different degrees. The turning points of PPV could be found between two curves of different temperatures, and the location of the turning point has special significance. When the detonation distance of the protective project is less than that of the turning point, the impact of blasting vibration under high temperature is stronger; otherwise, the condition of low temperature is not helpful for the safety of the protective project. The mathematical description of the detonation distance at the turning point is as follows:

$$
R^{*}=Q^{1 / 3}\left(\frac{K_{1}}{K_{2}}\right)^{\left(1 / \alpha_{1}-\alpha_{2}\right)}
$$

If the PPV distribution of $20^{\circ} \mathrm{C}$ was used as the benchmark, the comparison of the detonation distance versus the maximum charge weight per delay at the turning point is shown in Figure 13.

It can be seen that the special detonation distance is larger when the rock mass is in the temperature state of $-15^{\circ} \mathrm{C}$, and the result of the rock mass in $-5^{\circ} \mathrm{C}$ takes second place, while that of the rock mass under $-10^{\circ} \mathrm{C}$ is least. When the maximum charge weight per delay is $40 \mathrm{~kg}$, the special detonation distance of the rock mass under $-5^{\circ} \mathrm{C},-10^{\circ} \mathrm{C}$, and $-15^{\circ} \mathrm{C}$ is $29 \mathrm{~m}, 17 \mathrm{~m}$, and $35 \mathrm{~m}$, respectively. Results 


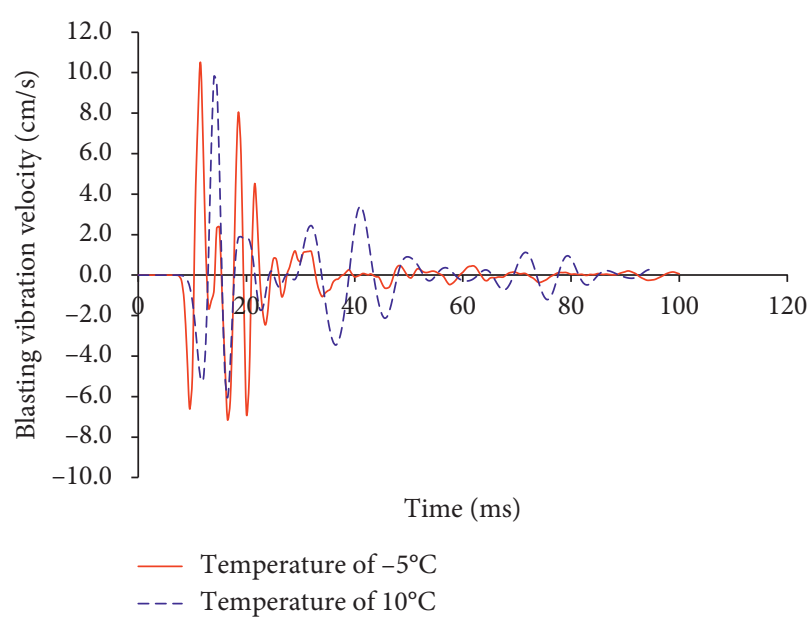

(a)

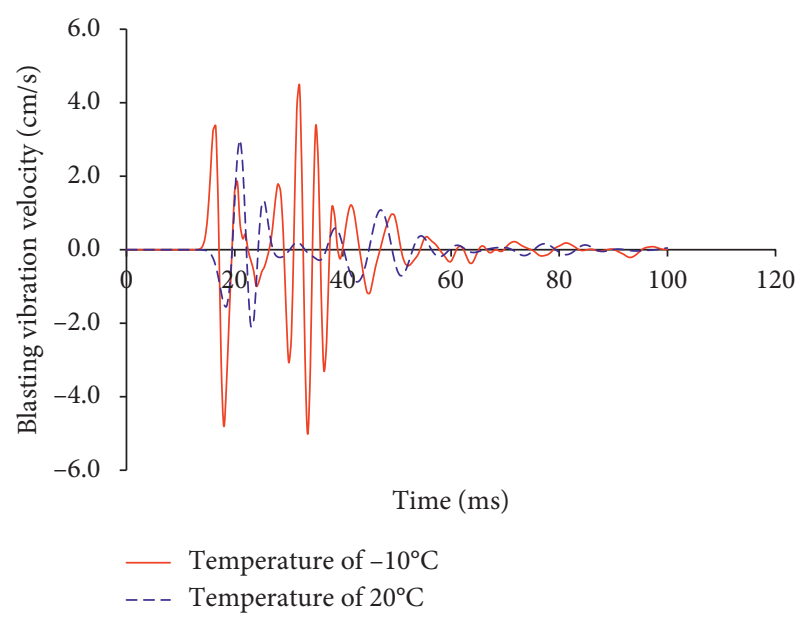

(c)

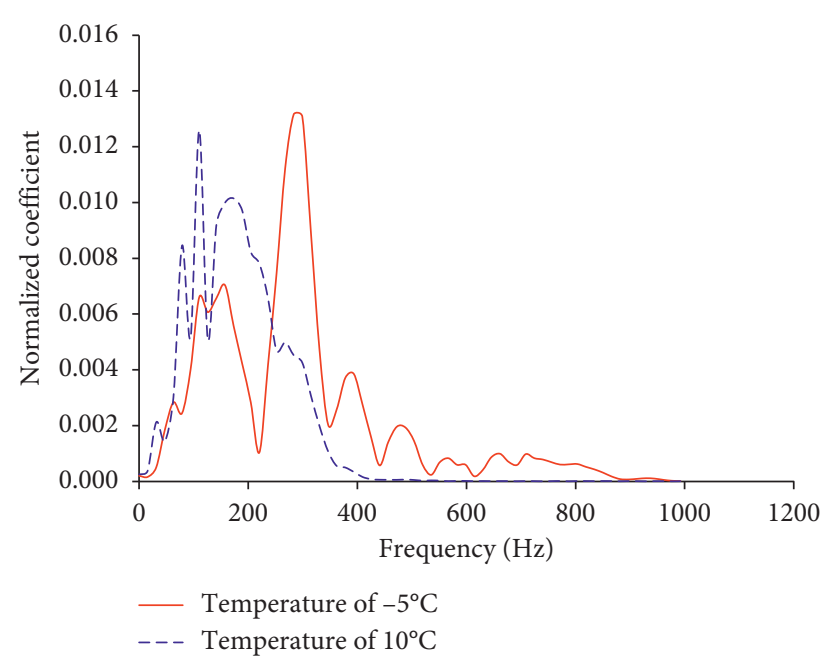

(b)

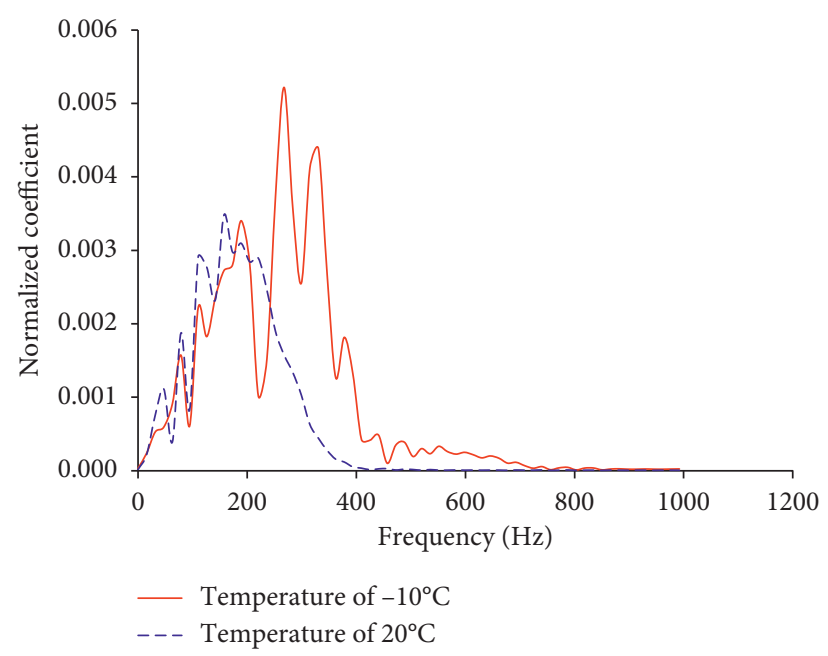

(d)

Figure 9: Comparison examples of blasting vibration waveform in the conventional and frozen rock mass. (a) Vibration waveform example I. (b) Frequency example I. (c) Vibration waveform example II. (d) Frequency example II.

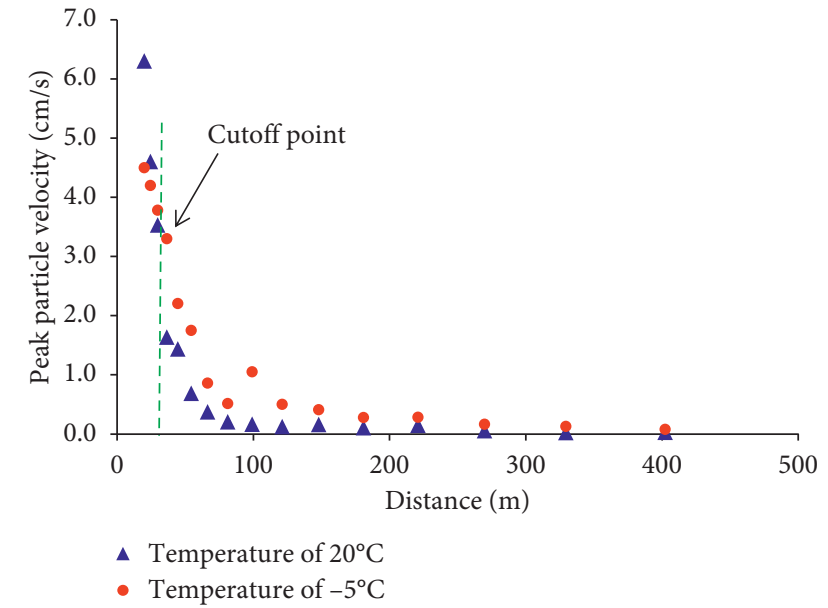

Figure 10: Distribution of PPV versus detonation distance at the temperature of $-5^{\circ} \mathrm{C}$ and $-20^{\circ} \mathrm{C}$. demonstrate the special detonation distance does not increase with the decrease of temperature strictly. For the same temperature condition, the special detonation distance increases with the maximum charge weight per delay and the relationship obeys the logarithmic function. As the locations of the turning point and protective project are important during the blasting vibration control process, the effect of temperature and the maximum charge weight per delay should be both taken into consideration.

\section{Numerical Simulations of the Response and Control in the Structure under Cold Condition}

Investigation of rock blasting and its effects by scaled or full size experiment is very expensive and time-consuming. On the other hand, numerical method, derived from sound mechanical principles and validated against experimental data, indicates a promising approach to study the response and control in the structure under cold condition. 


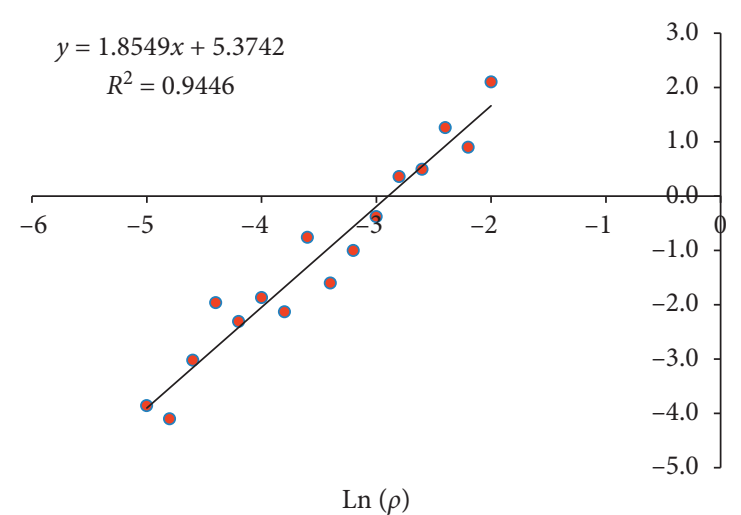

(a)

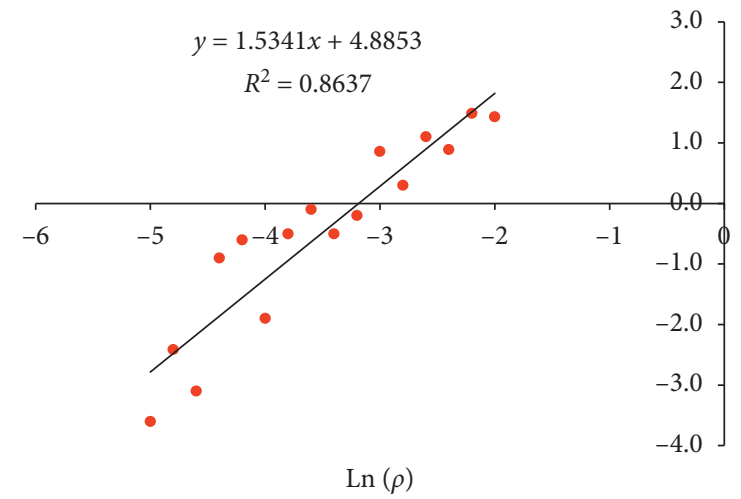

(c)

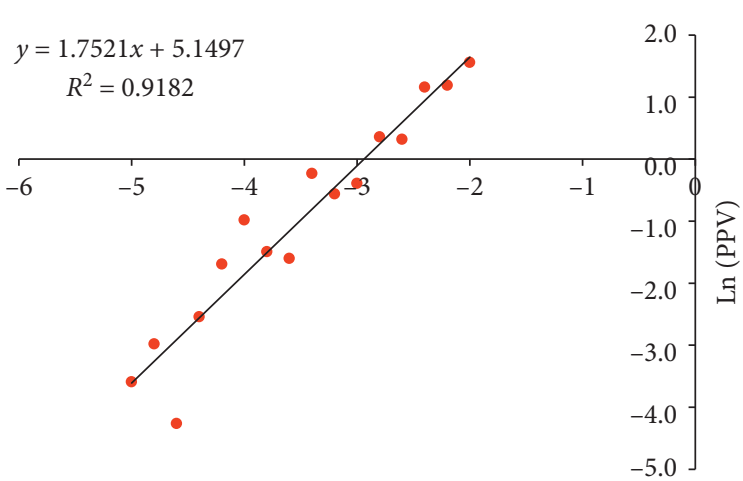

$\operatorname{Ln}(\rho)$

(b)

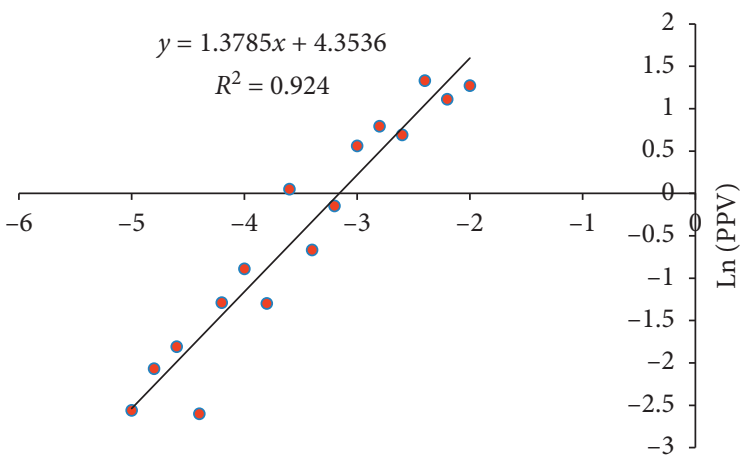

$\operatorname{Ln}(\rho)$

(d)

Figure 11: Blasting attenuation law curves of different temperatures in rock mass. (a) Temperature of $10^{\circ} \mathrm{C}$. (b) Temperature of $-5^{\circ} \mathrm{C}$. (c) Temperature of $-10^{\circ} \mathrm{C}$. (d) Temperature of $-5^{\circ} \mathrm{C}$.

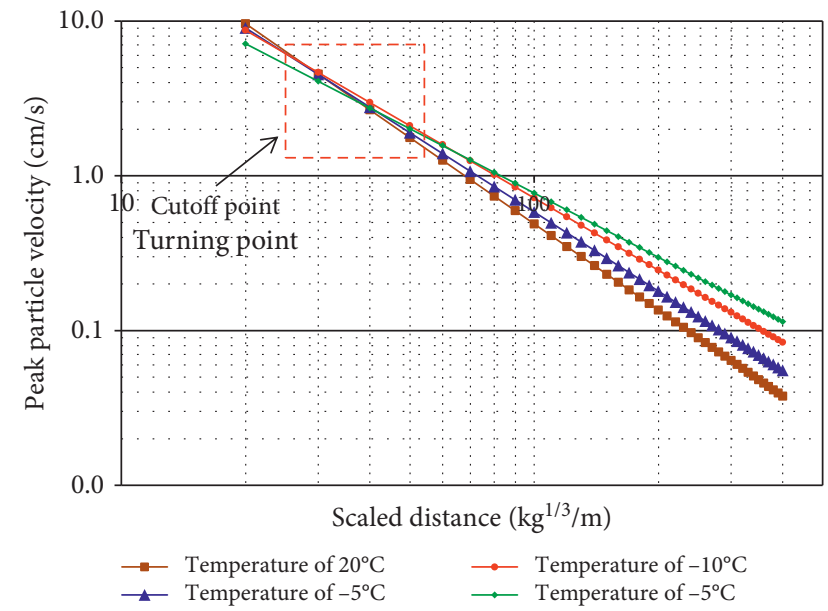

Figure 12: Comparison of the blasting attenuation law curves and the schematic of the turning point.

\subsection{The Numerical Tool and Equivalent Freezing Simulation} Method. To achieve a better understanding of this process, numerical simulation was implemented based on the case study of the foundation excavation of Fengman hydropower station. Figure 14 shows the developed numerical model to study the blast wave propagation in rock and structure. The model size was set to be $130 \mathrm{~m}$ long, $30 \mathrm{~m}$ wide, and $100 \mathrm{~m}$ high. A fine grid of elements is used in the vicinity of the charge to increase the simulation accuracy. The grid increases in size away from the charge, but it should be shorter than $1 / 8 \sim 1 / 10$ of the wavelength in general to properly reduce any wave distortions [23]. There are a total of 317,456 elements and 428,532 nodes in the numerical simulation. The source of the explosive is represented by a detonating cord containing a core load of emulsion explosive. Table 1 shows the used JWL equation of state parameters of the explosive. The contact setting of LS-DYNA was employed in the joint simulation. Nonreflecting boundaries are applied to the other surfaces, except the top surface which has the free boundary condition.

The water in the discontinuities in the rock mass close to the surface may change into ice under the low temperature. The numerous numbers of discontinuities in a rock mass in terms of the cracks and joints and their properties such as positions, orientations, strength, and stiffness are impossible to be known exactly, so the equivalent material property approach still remains the most popular and valuable means in practice in engineering. In the present study, the equivalent freezing simulation method was proposed, and detailed steps are as follows: firstly, the frozen depth and the temperature were determined according to the meteorological data; secondly, the physical-mechanical parameters of the frozen rock mass were obtained according to the laboratory test; and thirdly, the elements of the frozen layer in the numerical model were set as the frozen rock mass by 


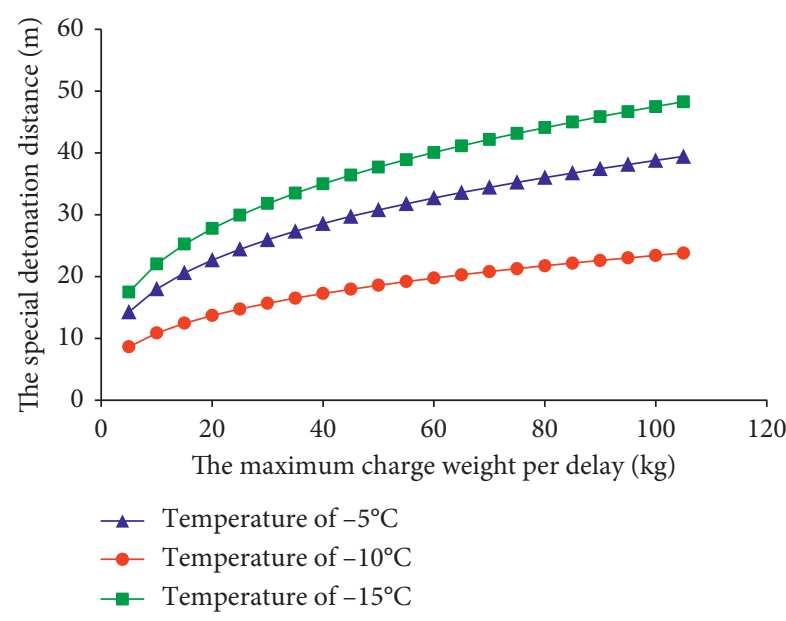

FIGURE 13: Detonation distance versus the maximum charge weight per delay at the turning point.

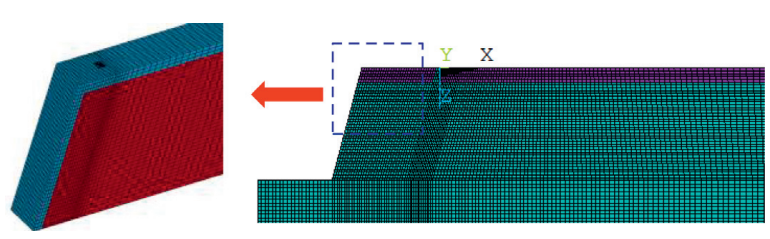

FIGURE 14: Numerical model to study the blast wave propagation.

adopting the different physical-mechanical parameters. Based on the laboratory test of the longitudinal wave velocity in the frozen rock mass, the elasticity modulus could be determined with equation (2). The density could be obtained directly with laboratory test. Table 2 shows the material constants for the constitutive model. Figure 15 plots the elasticity modulus increment versus the porosity in the frozen sandstone.

$$
E=\frac{V_{p}^{2} \rho(1+\mu)(1-2 \mu)}{(1-\mu)} .
$$

To reveal the rationality of this equivalent simulation approach, the comparison between the site experiment and numerical simulation was implemented. Figure 16 plots the comparison results of vibration waveform and the attenuation law.

It can be seen that the waveform of numerical simulation matches well with that of test results. The development process of blasting vibration waveform is quite similar, and the error of PPV is within 8\%. The attenuation law of PPV was also comprised of different conditions. As can be seen from Figure 16, the numerical curves of blasting attenuation law agree well with that of the site experiment when the temperatures are $-5^{\circ} \mathrm{C}$ and $20^{\circ} \mathrm{C}$. The error of the attenuation parameters of $K$ and $\alpha$ are both within 6\%. Results demonstrate that the numerical results agree well with the experiment data, which indicates that the equivalent simulation approach could predict the blasting vibration of the frozen rock mass under blasting.
4.2. The Determination of the Maximum Charge Weight per Delay in the Frozen Rock Mass. Numerical simulation could reproduce more kinds of working conditions compared with the site experiment. Figure 17 plots the relationship of attenuation coefficients such as $K$ and $\alpha$ versus the frozen depth, respectively, while the results of site experiment were also used as the comparison.

It can be seen that the linear relationship between the attenuation parameters and frozen depth could be obviously found, and the coefficient of correlation could reach as much as 0.9 . The mathematical descriptions are shown in equations (3) and (4). Results demonstrate that as the frozen depth of the rock mass increases, the attenuation parameters of $K$ and $\alpha$ decrease obviously, which indicates that the blasting seismic wave in rock mass attenuates slower after the rock mass was frozen. It can also be seen that the results of numerical simulation match well with that of site experiments.

$$
\begin{gathered}
K_{2}=0.87 K_{1}-65 d, \\
\alpha_{2}=\alpha_{1}-0.30 d .
\end{gathered}
$$

During the engineering practice, the maximum charge weight per delay is one of the most important indexes during the blasting vibration control. Usually, it could be determined with equation. (5). But when the rock mass was in the frozen state, the attenuation parameters of $K$ and $\alpha$ change obviously, which indicates that maximum charge weight per delay needs to be taken seriously. The description of the maximum charge weight per delay of the rock mass in the frozen state is shown in equation (6).

$$
\begin{aligned}
& Q=R^{3}\left(\frac{V}{K}\right)^{3 / \alpha}, \\
& Q=\frac{R^{3} V^{3 /\left(\alpha_{1}-0.30 d\right)}}{\left(0.87 K_{1}-64 d\right)^{3 /\left(\alpha_{1}-0.30 d\right)} .}
\end{aligned}
$$

Assuming that the vibration safety control standard of the building remains unchanged after the rock mass was frozen, the maximum charge weight per delay of the rock mass in the frozen state could be determined according to the above mathematical descriptions. Figure 18 plots the comparison of $Q_{\max }$ when the rock mass was in conventional and frozen states.

It can be seen that the maximum charge weight per delay change is less than that of conventional condition. The relationship between the frozen depth and the maximum charge weight per delay presents as the parabola characteristics. A minimum charge weight per delay could be found when the frozen depth is about $1.8 \mathrm{~m}$. If the frozen depth is less than $1.8 \mathrm{~m}$, the allowed charge weight per delay decreases with the frozen depth; otherwise, the maximum charge weight per delay increases with the frozen depth. Results demonstrate that the maximum charge weight per delay was limited more strictly during the frozen condition, and a most adverse depth exists. So it is necessary to pay 
TABLE 1: JWL equation of state parameters of the explosive used.

\begin{tabular}{lcccccccc}
\hline Density $\left(\mathrm{kg} / \mathrm{m}^{3}\right)$ & $\mathrm{VoD}(\mathrm{m} / \mathrm{s})$ & $P_{\mathrm{CJ}}(\mathrm{GPa})$ & $A(\mathrm{GPa})$ & $B(\mathrm{GPa})$ & $R 1$ & $R 2$ & $\omega$ & $E 0(\mathrm{GPa})$ \\
\hline 1000 & 3600 & 3.24 & 220 & 0.2 & 4.5 & 1.1 & 0.35 & 4.2 \\
\hline
\end{tabular}

VoD: velocity of detonation.

TABLE 2: Material constants for the constitutive model.

\begin{tabular}{|c|c|c|c|c|c|c|c|c|c|}
\hline Classes & $\begin{array}{l}\text { Density } \\
\left(\mathrm{kg} / \mathrm{m}^{3}\right)\end{array}$ & $\begin{array}{c}\text { Elasticity } \\
\text { modulus } \\
(\mathrm{GPa})\end{array}$ & $\begin{array}{l}\text { Poisson's } \\
\text { ratio }\end{array}$ & $\begin{array}{l}\text { Dynamic } \\
\text { tensile } \\
\text { strength } \\
(\mathrm{MPa})\end{array}$ & $\begin{array}{c}\text { Damage } \\
\text { constants } k\end{array}$ & $\begin{array}{c}\text { Damage } \\
\text { constants } m\end{array}$ & $\begin{array}{c}K_{I C} \\
\left(\mathrm{MN}^{*} * \mathrm{~m}^{-3 /}\right. \\
2)\end{array}$ & $\begin{array}{c}\text { Damage } \\
\text { constants } \lambda \\
(\mathrm{kg} / \mathrm{J})\end{array}$ & $\begin{array}{c}\text { Material } \\
\text { constants } \\
(\beta)\end{array}$ \\
\hline $\begin{array}{l}\text { Rock } \\
\text { mass }\end{array}$ & 2530 & 25 & 0.23 & 2 & $2.33 * 1024$ & 7 & 0.92 & 0.0001 & 0.5 \\
\hline
\end{tabular}

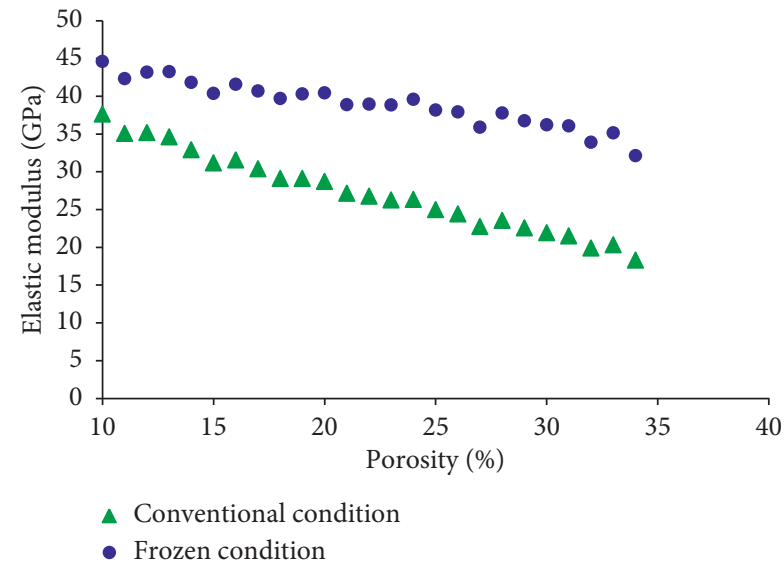

FIGURE 15: Elastic modulus increment versus the porosity in the frozen sandstone.

more attention to the control of the maximum charge weight per delay when the rock mass is in the frozen state.

\subsection{Comparison of the Dynamic Response of the Project of} Frozen and Conventional Conditions. The attenuation characteristic of blasting seismic wave is changed obviously in the frozen rock mass, which also affects the dynamic response of structures. To investigate the blasting vibration characteristic in the structure, the comparison of the structure response of blasting in frozen and conventional conditions was implemented with numerical simulation. Figure 19 plots the schematic diagram of the numerical structure model.

Figure 20 plots the comparison of the maximum principal stress, the minimum principal stress, the radial blasting vibration velocity, and the resultant blasting vibration velocity, respectively.

It can be seen that the stress in the frozen rock mass is larger than that in conventional condition. The maximum principal stress in frozen condition exceeds that in conventional condition by nearly $20 \%$, while that is about $10 \%$ for the minimum principal stress. The radial and resultant PPV of the frozen rock mass are also larger than those of the conventional rock mass. The resultant PPV of the conventional rock mass is only about $75 \%$ of that in frozen condition. Results demonstrate that the degree of dynamic response in structure under frozen condition is much stronger. This could be explained that the damping characteristic and geology were changed in the frozen rock mass, which makes the attenuation speed of blasting seismic wave slower.

During the engineering practice, the blasting vibration control standard was adopted as the threshold to limit the dynamic response in the structure within the allowed value. It is known that the tensile strength of the concrete is less than the compressive strength, so the tensile damage could be induced by blasting more easily. The blasting vibration control standard was determined based on the tensile stress control. The detailed steps are as follows: firstly, the numerical simulation of dynamic response in structure was implemented; then the relationship between the PPV at the foundation of structure and the maximum tensile stress of the structure was established; at last, when the maximum tensile stress is equal to the tensile strength, the corresponding PPV is the blasting vibration control standard. Figure 21 plots the comparison of the blasting vibration control standard under the cold and conventional conditions.

It can be seen that the typical linear relationship between the PPV and the maximum tensile stress could be both found under the conventional and frozen conditions. Results reveal that the blasting vibration control standard of the frozen rock mass is larger than that of conventional condition, which indicates that the frozen is beneficial for the blasting vibration safety control. But it should be pointed that the results do not mean the safety control of the blasting vibration in the frozen state is looser. As mentioned in Section 4.2, the maximum charge weight per delay in the frozen rock mass is obviously less than that in the conventional condition. Under the conventional condition, the blasting vibration control standard is $9.6 \mathrm{~cm} / \mathrm{s}$, but for the rock mass in the frozen state, that is $10.7 \mathrm{~cm} / \mathrm{s}$. During the blasting vibration safety control of the Fengman hydropower station reconstruction project, the blasting vibration control standard was determined as $10 \mathrm{~cm} / \mathrm{s}$. A total of $676 \mathrm{moni}-$ toring was completed, and no case of excessive measurement could be found. 


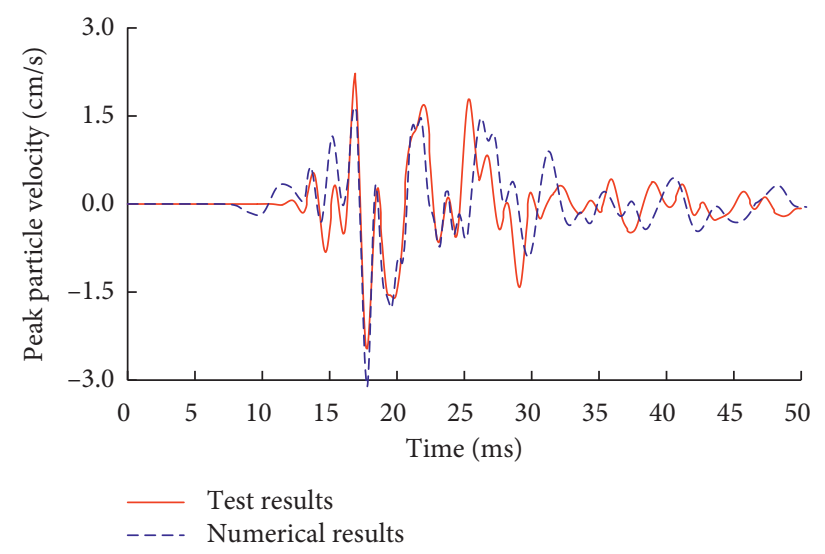

(a)

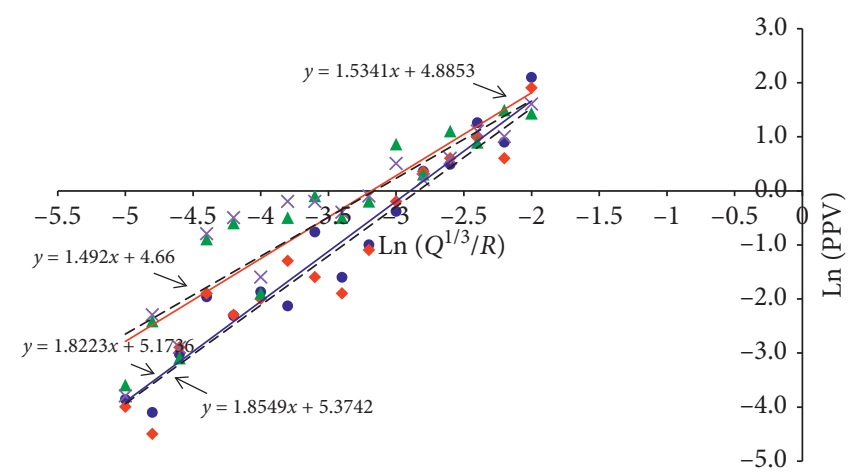

- Test results of $20^{\circ} \mathrm{C} \quad \Delta \quad$ Test results of $-5^{\circ} \mathrm{C}$

- Numerical results of $20^{\circ} \mathrm{C} \times$ Numerical results of $-5^{\circ} \mathrm{C}$

FIGURE 16: Comparison results of vibration waveform and the attenuation law.

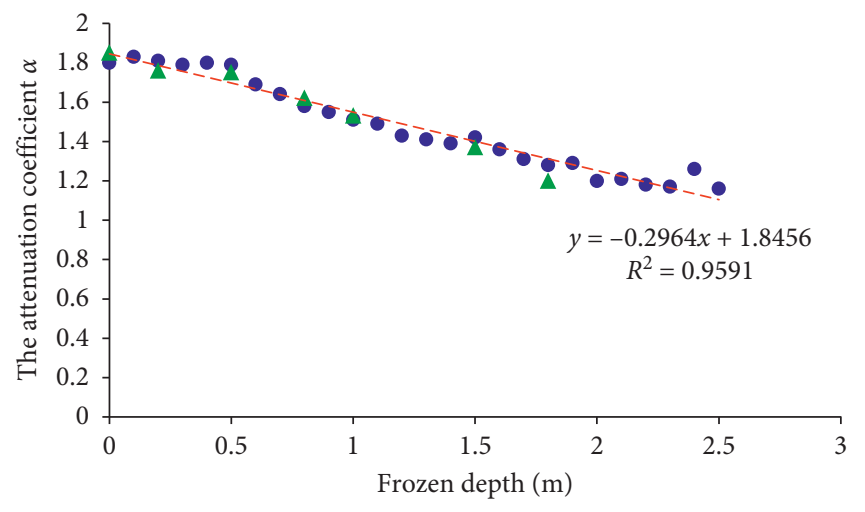

- Numerical simulation

- Site experiment

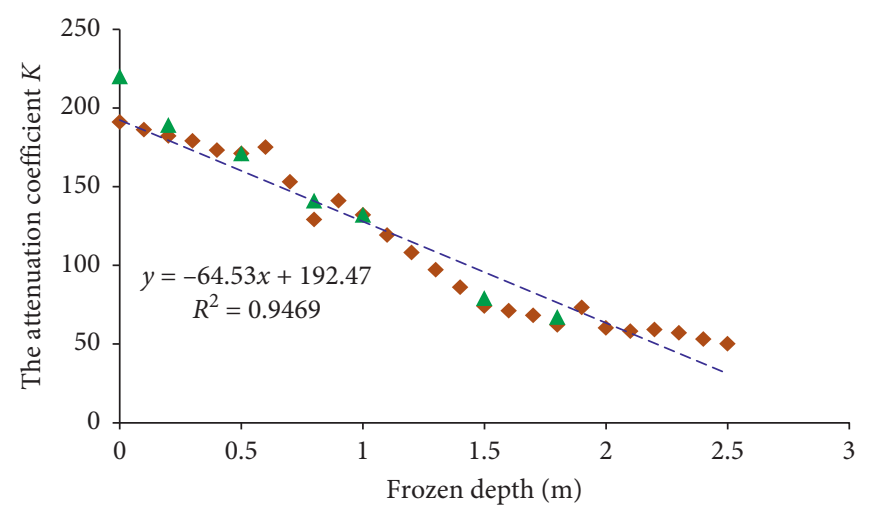

Numerical simulation

A Site experiment

(a)

(b)

Figure 17: Variation law of vibration attenuation parameters versus frozen depth. (a) Coefficient $\alpha$. (b) Coefficient $K$.

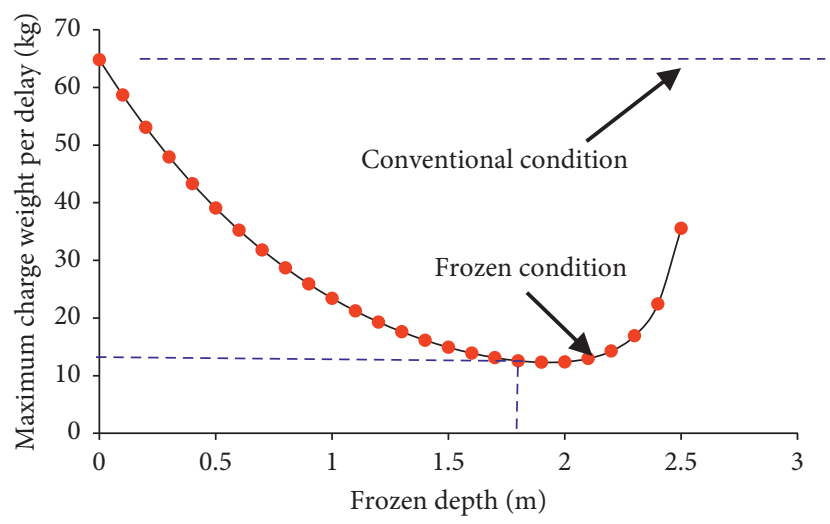

Figure 18: Comparison of $Q_{\max }$ when the rock mass was in conventional and frozen states. 

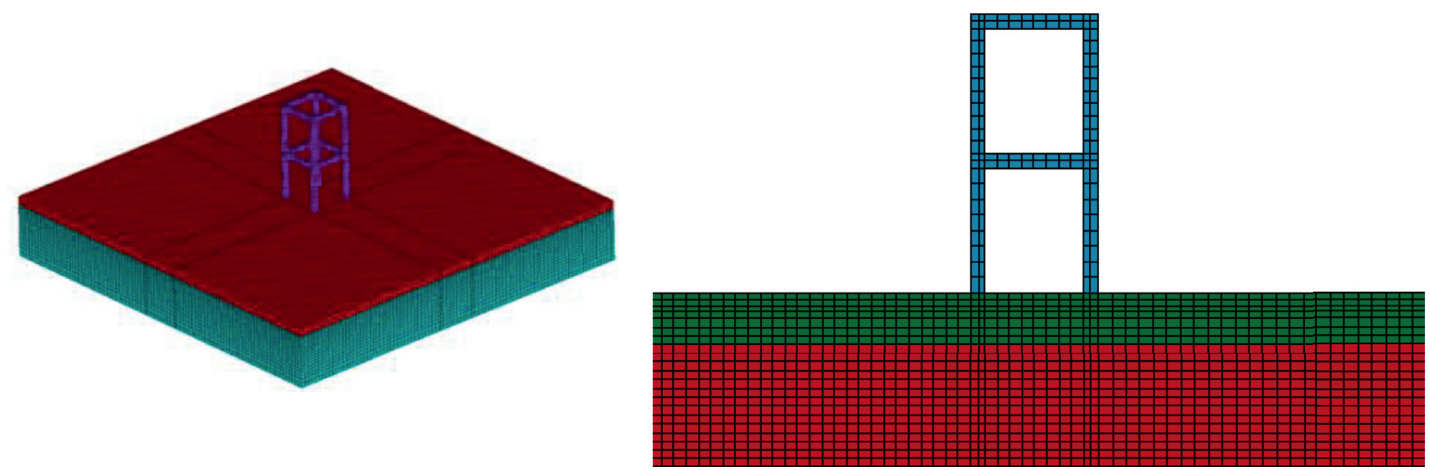

FIGURE 19: Schematic diagram of the numerical model for structural response analysis.

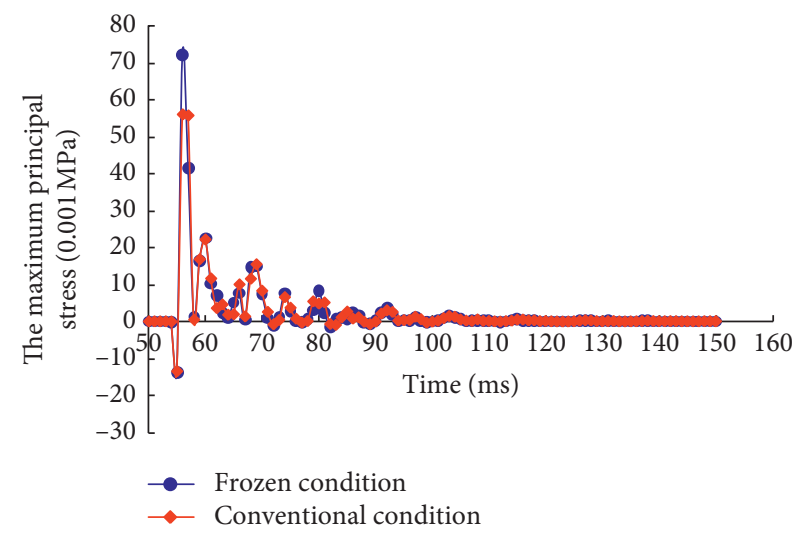

(a)

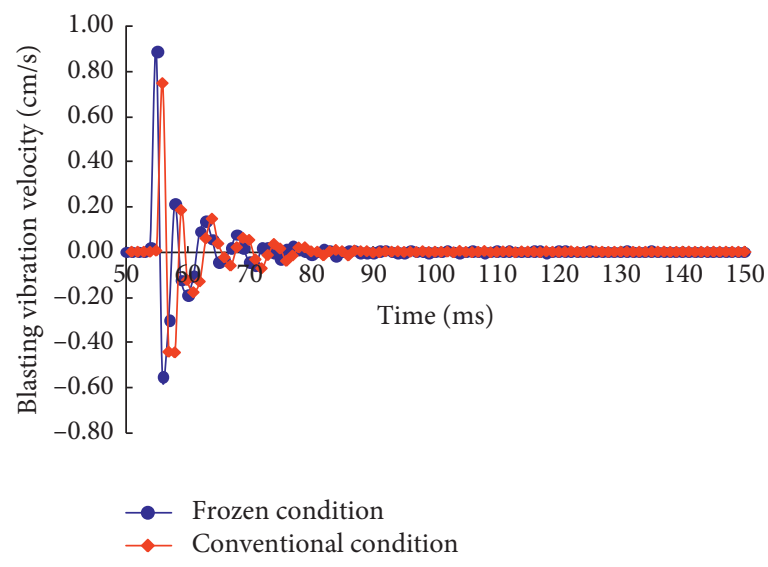

(c)

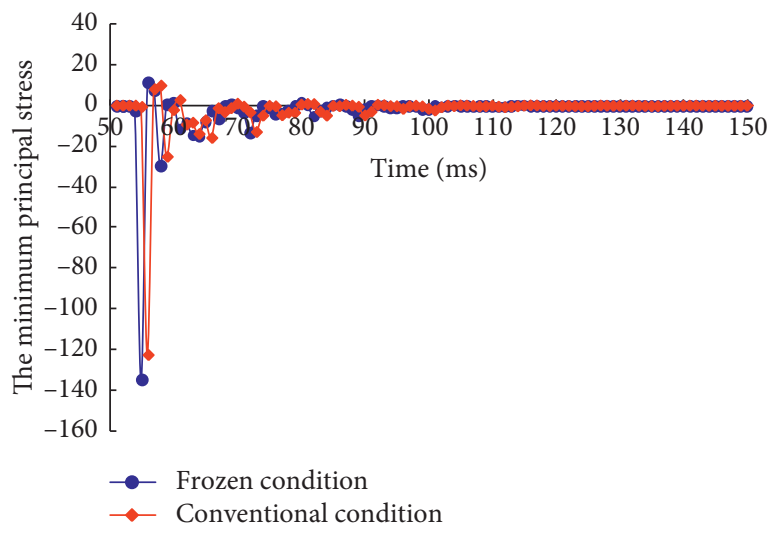

(b)

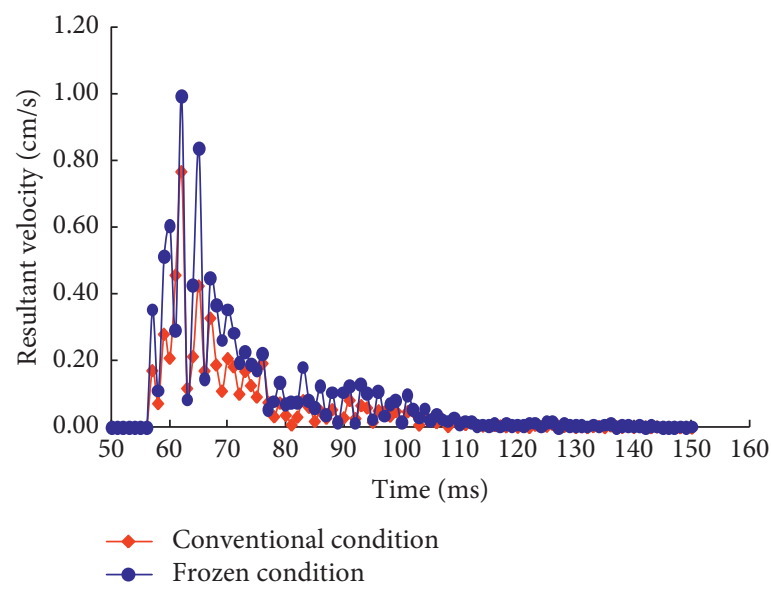

(d)

Figure 20: Comparison of the dynamic response in the frozen and conventional conditions. (a) The maximum principal stress. (b) The minimum principal stress. (c) The radial blasting vibration velocity. (d) The resultant blasting vibration velocity. 


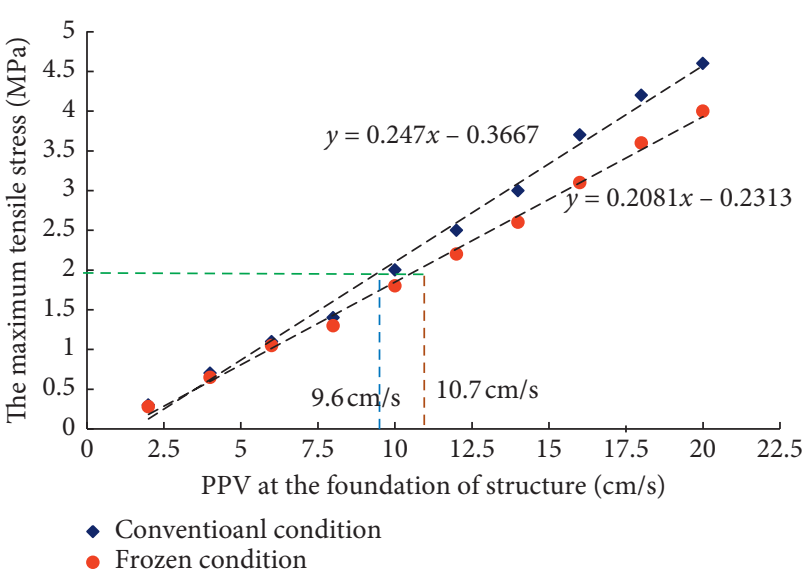

FIgURE 21: Comparison of the blasting vibration control standard under the cold and conventional conditions.

\section{Conclusions and Discussions}

This paper focuses on the blasting vibration control technology of the frozen rock mass under cold condition. Laboratory tests were implemented to investigate the change of mechanical performance for rock mass at the frozen, saturated, normal, and drying states. Then long-term monitoring of blasting vibration was implemented based on the blasting excavation of Fengman hydropower station reconstruction project in China to study the vibration attenuation law of the rock mass at different seasons. The blasting vibration declines slower in winter for the frozen rock mass. At last, numerical simulation of blasting vibration attenuation at different states was finished and suggestion of blasting vibration control of rock mass excavation under cold condition was proposed. The following conclusions and understandings may be drawn from the present study.

The longitudinal wave velocity in the frozen state is the biggest, and the wet saturated state takes the second place, while the natural state is third and the dry state is the least. The longitudinal wave velocity exceeds that of the natural state by about 10\% 35\% in the Fengman hydropower station area, and the difference between the frozen and natural state increases with the porosity obviously. The ability to conduct the stress waves is enlarged when the rock mass is frozen, especially in the rock medium with high porosity. The wave impedance increment and the porosity obey the exponential relationships obviously.

Results demonstrate the PPV attenuates slower when the rock mass is frozen, which indicates that the quality of the rock mass becomes better. The frequency of the blasting vibration in the frozen rock mass is also larger than that in the conventional condition. As the temperature decreases, the attenuation coefficient $K$ and $\alpha$ all decrease by different degrees. The obvious turning points of PPV could be found between different temperatures, where the change of the PPV relationship happens.

The equivalent freezing simulation method was proposed to simulate the response in the frozen rock mass and structures. Results demonstrate that the waveform of numerical simulation matches well with that of test results. The development process of blasting vibration waveform is quite similar and the error of PPV is within $8 \%$. The relationship of attenuation coefficients such as $K$ and $\alpha$ versus the frozen depth was determined, respectively. The linear relationship between the attenuation parameters and frozen depth could be obviously found, and as the frozen depth of the rock mass increases, the attenuation parameters of $K$ and $\alpha$ decrease obviously.

The maximum charge weight per delay and blasting vibration control standard were investigated in the frozen rock mass to control the blasting vibration. The relationship between the frozen depth and the maximum charge weight per delay presents as the parabola characteristics. The maximum charge weight per delay was limited more strictly during the frozen condition, and a most adverse depth exists. The degree of dynamic response in structure under frozen condition is much stronger. The blasting vibration control standard in the frozen condition is larger than that in the conventional condition. With the above control approaches, a total of 676 monitoring was completed in the Fengman reconstruction project, and no case of excessive measurement could be found.

\section{Data Availability}

The data supporting the results of this study can be obtained upon request to the corresponding author.

\section{Conflicts of Interest}

The authors declare that they have no conflicts of interest regarding the publication of this paper.

\section{Acknowledgments}

This work was supported by the Chinese National Natural Science Foundation (52079009) and the Central Scientific Research Operating Expenses (CKSF2019477/YT, CKSF2019193/YT). The authors wish to express their thanks to all supporters.

\section{References}

[1] O. Dogan, Ö. Anil, S. O. Akbas, E. Kantar, and R. Tuğrul Erdem, "Evaluation of blast-induced ground vibration effects in a new residential zone," Soil Dynamics and Earthquake Engineering, vol. 50, pp. 168-181, 2013.

[2] D. Park, B. Jeon, and S. Jeon, "A numerical study on the screening of blast-induced waves for reducing ground vibration," Rock Mechanics and Rock Engineering, vol. 42, no. 3, pp. 449-473, 2008.

[3] G. Ma, H. Hao, and Y. Lu, "Modelling damage potential of high-frequency ground motions," Earthquake Engineering \& Structural Dynamics, vol. 32, no. 10, pp. 1483-1503, 2003.

[4] J. H. Yang, J. H. Dai, C. Yao, S. H. Jiang, C. B. Zhou, and Q. H. Jiang, "Estimation of rock mass properties in excavation damage zones of rock slopes based on the Hoek-Brown criterion and acoustic testing," International Journal of Rock Mechanics and Mining Sciences, vol. 126, 2020.

[5] H. R. M. Azizabadi, H. Mansouri, and O. Fouché, "Coupling of two methods, waveform superposition and numerical, to 
model blast vibration effect on slope stability in jointed rock masses," Computers and Geotechnics, vol. 61, pp. 42-49, 2014.

[6] H. Ak, M. Iphar, M. Yavuz, and A. Konuk, "Evaluation of ground vibration effect of blasting operations in a magnesite mine," Soil Dynamics and Earthquake Engineering, vol. 29, no. 4, pp. 669-676, 2009.

[7] I. A. Onederra, J. K. Furtney, E. Sellers, and S. Iverson, "Modelling blast induced damage from a fully coupled explosive charge," International Journal of Rock Mechanics and Mining Sciences, vol. 58, pp. 73-84, 2013.

[8] E. Wang and A. Shukla, "Analytical and experimental evaluation of energies during shock wave loading," International Journal of Impact Engineering, vol. 37, no. 12, pp. 1188-1196, 2010.

[9] J. Yang, J. Cai, C. Yao, P. Li, Q. Jiang, and C. Zhou, "Comparative study of tunnel blast-induced vibration on tunnel surfaces and inside surrounding rock," Rock Mechanics and Rock Engineering, vol. 52, no. 11, pp. 4747-4761, 2019.

[10] R. E. Goodman and D. S. Kieffer, "Behavior of rock in slopes," Journal of Geotechnical and Geoenvironmental Engineering, vol. 126, no. 8, pp. 675-684, 2000.

[11] S. A. Ashford, N. Sitar, J. Lysmer, and N. Deng, "Topographic effects on the seismic response of steep slopes," Bulletin of the Seismological Society of America, vol. 87, pp. 701-709, 1997.

[12] A. Kesimal, B. Ercikdi, and F. Cihangir, "Environmental impacts of blast-induced acceleration on slope instability at a limestone quarry," Environmental Geology, vol. 54, no. 2, pp. 381-389, 2007.

[13] M. Chen, W. B. Lu, P. Yan, and C. B. Zhou, "New method for dynamic stability analysis of rock slope under blasting vibration based on equivalent acceleration and Sarma method," Canadian Geotechnical Journal, vol. 51, pp. 441-448, 2013.

[14] W.-B. Lu, Y.-G. Hu, J.-H. Yang, M. Chen, and P. Yan, “Spatial distribution of excavation induced damage zone of high rock slope," International Journal of Rock Mechanics and Mining Sciences, vol. 64, pp. 181-191, 2013.

[15] J. Toraño, R. Rodríguez, I. Diego, J. M. Rivas, and M. D. Casal, "FEM models including randomness and its application to the blasting vibrations prediction," Computers and Geotechnics, vol. 33, no. 1, pp. 15-28, 2006.

[16] W. B. Lu, J. H. Yang, M. Chen, and C. B. Zhou, "An equivalent method for blasting vibration simulation," Simulation Modelling Practice Theory, vol. 19, pp. 2050-2062, 2011.

[17] C. Kuzu, "The importance of site-specific characters in prediction models for blast-induced ground vibrations," Soil Dynamics and Earthquake Engineering, vol. 28, no. 5, pp. 405-414, 2008.

[18] T.-C. Pan, K. S. Goh, and K. Megawati, "Empirical relationships between natural vibration period and height of buildings in Singapore," Earthquake Engineering \& Structural Dynamics, vol. 43, no. 3, pp. 449-465, 2014.

[19] Y. Lu, H. Hao, G. Ma, and Y. Zhou, "Simulation of structural response under high-frequency ground excitation," Earthquake Engineering \& Structural Dynamics, vol. 30, no. 3, pp. 307-325, 2001

[20] I.-S. Ha, "Estimation of shear wave velocity of earth dam materials using artificial blasting test," Soil Dynamics and Earthquake Engineering, vol. 55, pp. 120-129, 2013.

[21] F. Jalayer, D. Asprone, A. Prota, and G. Manfredi, "Multihazard upgrade decision making for critical infrastructure based on life-cycle cost criteria," Earthquake Engineering \& Structural Dynamics, vol. 40, no. 10, pp. 1163-1179, 2011.

[22] P. Li, W. B. Lu, X. X. Wu, M. Chen, P. Yan, and Y. G. Hu, "Spectral prediction and control of blast vibrations during the excavation of high dam abutment slopes with milliseconddelay blasting," Soil Dynamics and Earthquake Engineering, vol. 94, pp. 116-124, 2017.

[23] R. L. Kuhlemeyer and J. Lysmer, "Finite element method accuracy for wave propagation problems," Journal of the Soil Mechanics and Foundations Division, vol. 99, no. 5, pp. 421427, 1973. 